\title{
III. Geburtshilflich-gynäkologische Mitteilungen aus anderen
} medizinischen Gesellschaften

$<3 / 8$ eifswald. 14.1. (Dtsch. med. Woch. 31. III. S. 370.) Hoehne: Über Blutungen bei vorgerückter Gravidität sowie intra partum und über ihre Behandlung. - Stephan: Über den Metranoikter als Dilatationsinstrument und seine anatomische Wirkungsweise. - 29. I. Liß: Zur Caseosan-therapie in Geburtshilfe und Gynäkologie.

Jena. 2. II. (Munch, med. Woch. 9. IV. S. 438.) Schuchardt: Men-struationspsychosen.

Prag. 18. XI. (Dtsch. med. Woch. 7. IV. S. 404.) Porger: Kystadenom dee rechten Ovarium. Rezidiv nach Operation. - Ederer: Nachweis der Vollständigkeit der Plazenta.

Medizinische Gesellschaft Berlin. 2. III. (Dtsch. med. Woch. 24. III. S. 349.) 0. Schlesinger: Zur Frage der klimakterischen Blutdrucksteigerung.

Köln. Februar. (Dtsch. med. Woch. 24. III. S. 340.) Budde: Über die Genese der Teratome. Französische Gesellschaft für Krebsstudien. 15. XI. (Gynéc. obst. III. Nr. 4. S. 277.) H.

Hartmann u. Renaud: Brustkrebs; histologisch Epithe-lion und Tuberkulose. -Lecène: Epithelioma mucosae corporis mit Zylinder und Pflasterepithel.

Medizinisch-bioiogische Gesellschaft Montpellier und des südlichen Languedoc. (Gynéc. obstet. III. H. 4. S. 279.) Estor, Grynfeld u. Aimer, Fibromyoma ovarii einer 52 jähr. Pat. - Roux u. Vinon: Epithelioma canaliculare der Brust. - Mados: Agglutinatio colli. - Rvume: Toxischinfektiöses Sjmdrom einer Gravida - Symptom einer Encephalitis lethargica.- Vinon u. Roux: Große Uteruszyste mit torquirtem Stiel. - Gueit u. Pouíeí: Wiederaufnahme der Milchernährung nach einmonatlicher Unterbrechung.

Gesellschaft der Ärzte Wiens. 11. III. (Wien. klin. Woch. Nr. 12. S. 138.) Sgalitzer: Die Zystographie.

Verein deutseher Ärzte in Prag. (Med. Klin. 20. III. S. 365.) H. H. Schmid: Darmverletzung bei Unterbrechung einer vermeint lichen Schwangerschaft.

Ärztlicher Verein Frankfurt. 1. XL (Munch, med. Woch. 25. Ill, S. 377.) Síppel: Heterotope Zwillingsschwangerschaft. Bespr.: Goetze. Schef $\uparrow$ er, Flesch.

Ärztlicher Bezirksverein Erlangen. 23. II. (Munch, med. Woch. 25. III. S. 379.) Seiíz-Winíz: 1. Klinische Erfahrungen in der Strahlenbehandlung des Karzinom. - 2. Über die Verkupferung der Karzinom als Sensi-bilisierungsmethode.

Medizinische Gesellschaît Magdeburg-. 11. XI. (Munch, med. Woch. 18. III. S. 347.) Penkert:

Haemata vulvae (Notzuchtsversuch). - Karzi-

300

Buchbesprechungeii.

noma Vulvae mit Durchbruch ins Rektum. - Auffallend großer Blasen-stein, operativ gewonnen. - Lymphosarkomatose.

Berliner medizinische Gesellschaft. 16. III. (Berl. klin. Woch. 11. IV. S. 363.) Vogel:

Endoventrale Operation tiefsitzender Harnleitersteine. Bespr.: A. Freudenberg. - Hamburger: 
Arzt und Bevölkerungspolitik. Bespr.: A. Freudenberg. - 23. III. Bornstein, Poll, Grotjahn, Lazarus, Theilháber.

Medizinische Gesellschaft Jena. 3. XI. (Munch, med. Woch. 1. IV. S. 406.) Engelhorn: AbtreibiingsversiTche mit nicht ganz alltäglichem Verlauf.

Medizinische Gesellschaft Leipzig. 30. XI. (Munch, med. Woch. 1. IV. S. 406.) Kleinschmídt:

Blasenspaltenoperation. - 14. XI. P. Zweifel: Neue Gesichtspunkte zur Verhütung des

Kindbettfiebers. Bespr.: Payr, Thíes.

Wissenschaftlicher Verein Stettin. 8. II. Munch, med. Woch. 1. IV. S. 109. Sandhop:

Kindbettfieber und Abort vom Standpunkt des Sozial-hygienikers. Bespr.: Schwarzwäller,

Davidson, Lichtenauer.

Mediziniseh-naturwissenschaftlicher Verein Tubingen. 22. XI. (Munch, med. Woch. 22. XL S. 416.) Barlram: Über Behandlung des Puerperal-fiebers mit menschlichem Serum. - A. Mayer: Die diagnostische und therapeutische Bedeutung des Pneumoperitoneum. - Die operative Erweiterung der Schrumpfblase. - E. Vogt: Röntgenuntersuchungen der inneren Organe des Neugeborenen. - Das Steinkind von Weißbach.

Heidelberg. 27. VI. (Berl. klin. Woch. 25. IV. 20. S. 422.) Klein-schmidt: Zur therapeutischen Verwendung des Pneumoperitoneum.

Kiel. 1. VII. 20. (Berl. klin. Woch. 25. IV. S. 423.) Stoeckel: Über Schnittentbindung bei Placenta praevia. Bespr.: Holzapfel. - Bauereisen : 1. Junges Ei in situ. 2. Myom und Pyonephrose. Bespr.: Stoeckel, Jores. - Meyer: Eigenmilchinjektion zur Hebung der Stillfähigkeit. Bespr.: Stoeckel, Jores, v. Stark, Holzapfel, Behn, Spiegel, Schackwítz. - 15. VII. Becker: Kompression der Aorta abdominalis zur Stillung geburtshilflicher Blutungen. Bespr.: zur Verth, Anschütz, Stoeckel. - - Bauereisen: Demonstration eines Falles von Hebotomie. Breslau. 26. XL 20. (Berl. klin. Woch. 25. IV. S. 426.) Herfarth: Ver-größerung der Portio bei totgeborenem ausgetragenem Mädchen. Bespr.: Küstner.

IV. Buchbesprechungen.

Rud. Th. v. Jaschke und 0. Pankow, Lehrbuch der Gebur ¿shilfe. 9. Auf-lage von „M. Runges Lehrbücher der Geburtshilfe und Gynäkologie”. Verlag Julius Springer, Berlin 1920.

Unter Wahrung ihrer persönlichen Anschauungen haben die beiden Autoren das längst vergriffene Lehrbuch der Geburtshilfe von M. Runye 